\title{
Meeting recommended dietary intakes in meal plans with $\geq 4$ servings of grain-based foods daily
}

\author{
Yasmine Probst* and Linda Tapsell \\ Smart Foods Centre, University of Wollongong, Wollongong, NSW 2522, Australia
}

Submitted 18 August 2011: Final revision received 7 February 2012: Accepted 26 August 2012: First published online 16 November 2012

\begin{abstract}
Objective: To develop meal plans using grain-based foods demonstrating how to incorporate wholegrain foods into a balanced diet for weight maintenance for different cuisines. The present study examines the ability of meal plans with $\geq 4$ grain-based servings daily to meet nutrient recommendations using lacto-ovo vegetarian and rice-based cuisines.

Design: Eighteen plans from each cuisine for three age brackets for both genders were developed. Plans aimed for $\geq 4$ servings of grain-based foods daily, with separate plans for all wholegrain, all refined-grain and half wholegrain-half refined-grain foods. Meal plans followed an isoenergetic approach and were designed to meet specific Australian nutrient reference values and serving sizes. Results: All plans met the Recommended Dietary Intake or Adequate Intake for targeted nutrients except for $\mathrm{Fe}$ in the rice-based meal plan for females aged $\geq 19$ years $(17 \mathrm{mg}$ ). In the plans for $14-18$ year and $\geq 19$ year age groups, four servings of grain-based foods could be accommodated. In the plans for 9-13 years, increasing the number of grain-based food servings to four reduced micronutrients levels delivered by the total diet. Specific food choices were made to ensure nutrient targets were met across each category for wholegrain and refined-grain plans. The major difference in nutrients between wholegrain and refined-grain foods was found in the vegetarian cuisine, where the meal plans containing whole grains produced on average $30 \%$ higher fibre (38-53 g) levels than those with refined grains $(27-40 \mathrm{~g})$.

Conclusions: With careful food selection, meal plans with $\geq 4$ servings of grainbased foods daily can meet nutrient reference values for lacto-ovo vegetarian and rice-based cuisines.
\end{abstract}

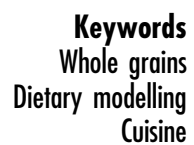

Cuisine
A significant body of research shows that a higher consumption of whole grains is associated with reduced risk of disease ${ }^{(1-3)}$. When considering refined grains, however, the strength of these associations is reduced and inconsistent ${ }^{(4,5)}$. For example, in the Atherosclerosis Risk in Communities Study, Steffen et al. found that consumption of refined-grain foods - median intake of two servings of grain-based foods daily - was associated with lower educational attainment, unhealthy behaviours and an unbalanced diet $(P<0 \cdot 001)^{(4)}$. At this level of consumption there was a trend for increased risk of disease compared with those who consumed 0.5 servings daily $(P<0 \cdot 001)$. On the other hand, Liu et al. found no evidence for the association between consumption of refined grains and risk of CVD in the Nurses' Health Study ${ }^{(5)}$.

One of the issues in research on grain-based foods, however, is the classification of wholegrain and refinedgrain foods. FFQ may not always differentiate wholegrain food items from refined-grain food items. For example, the term 'rice' often includes white rice, brown rice and wild rices; the latter two being whole grains.

The amount of a food also needs to be considered to determine this impact on health. Serving sizes for grainbased foods are inconsistent ${ }^{(6,7)}$. For example in Australia, reference standards for one serving of grain food is equivalent to two slices of bread $^{(8)}$, while it is equivalent to only one slice of bread in other published material ${ }^{(9)}$. To add to this problem, the Australian Guide to Healthy Eating $(\mathrm{AGHE})^{(10)}$ defines a sample serving of breads and cereals (including rice, pasta and noodles) as equivalent to $600 \mathrm{~kJ}$ or two slices ( $60 \mathrm{~g}$ ) of bread. When different types of grainbased foods from different cultural cuisines are considered these serving sizes create further analytical challenges.

The term 'cuisine' can be described as a set of foodrelated practices, particularly in relation to a cultural group. This concept covers not only particular types of foods eaten and their characteristic flavours, but also the symbolic and social contexts of eating, processing techniques, as well as the nutritional value of these foods ${ }^{(11)}$. 
A lacto-ovo vegetarian diet, for example, means choosing from a variety of grain-based foods, legumes, vegetables, fruit, milk and other dairy foods plus eggs, while avoiding other animal foods. A well-planned vegetarian diet can be lower in saturated fat, cholesterol and animal protein while still containing greater amounts of beneficial nutrients including carbohydrate, fibre, $\mathrm{Mg}$, folate and a variety of antioxidants ${ }^{(12)}$, although there may be risk of some nutrient deficiencies - Fe, Zn and long-chain $n-3$ fatty acids - if the diet is not well planned ${ }^{(12)}$. Another example is the Asian cuisine, characterised by the use of rice as a staple food. This cuisine has become increasingly common in Australia, with Chinese the predominant cultural influence through migration from South-East $\mathrm{Asia}^{(13)}$. Rice is consumed in many forms, from foods based on whole grains and refined grains, to noodles, desserts, congee and sake. Rice used in Asian cuisine is typically milled white rice and the milling process removes fibre, protein, $\mathrm{Fe}$, fat and $\mathrm{B}$ vitamins ${ }^{(14)}$.

Determining the impact of specific food choices within different cuisines requires consideration of meal structure, nutrient bioavailability and combinations of food and availability, as well as the overall appropriateness of nutrient composition of the daily or weekly intake. This appropriateness may be determined through the use of nutrient reference values ${ }^{(15)}$; however, a number of different values exist and should be used carefully and for the correct purpose.

The Recommended Dietary Intake (RDI) value should be used for assessing the adequacy of diets for individuals, not for groups of people. When an RDI is unavailable, an Adequate Intake (AI) value may also be provided based on determined approximations and intakes for individuals. Values above these approximations are considered to have a low probability of an inadequate intake of the particular nutrient. Similarly for groups, the prevalence of this inadequacy may be assessed. The Acceptable Macronutrient Distribution Range (AMDR) allows for the adequacy of intake to be calculated for different age groups and genders ${ }^{(15)}$.

Finally, to determine whether a dietary pattern might achieve nutrient reference values, good-quality food composition data are required. The data allow meal plans to be developed using specific food combinations and meal patterns.

The aim of the present study was to examine the ability of meal plans designed with $\geq 4$ grain-based servings daily (refined and whole grains) to meet RDI, AI and $\mathrm{AMDR}^{(15)}$ values within a weight management model for two different cuisine types, namely lacto-ovo vegetarian and rice-based (quasi-Asian). The objectives of the meal plans were to include a healthy balanced diet for both cuisine types, including all major food groups and their alternatives, for males and females aged 9 years and older. Australian guidelines suggest a minimum of four grain-based servings are required each day for optimal health and well-being, and to date Australia does not have a guideline stating the minimum amount of whole grains to be included in the diet for optimal health as is seen in the USA, Canada and some European countries. Furthermore, the term 'grains' may include both wholegrain and refined-grain foods. Therefore, the study sought to test the effect if all of these four servings were whole grains or refined grains or a combination of the two. Wholegrain meal plans were developed to test whether four wholegrain servings could be included each day. These meal plans were compared with refined grain and half refined grain/half wholegrain meal plans.

\section{Methods}

Thirty-six $7 \mathrm{~d}$ meal plans were developed in FoodWorks version 5.001324 (Xyris Software Ltd, Highgate Hill, Australia) using 1999 AUSNUT and 2001 AusFoods databases ${ }^{(16)}$ with the aim of achieving as many of the RDI and AI values as possible ${ }^{(17)}$ using the serving sizes and number of servings recommended by the National Health and Medical Research Council in Food for Health: $A$ Guide to Healthy Eating ${ }^{(7)}$ and the $\mathrm{AGHE}^{(10)}$ where additional information was required. Meal plans were based on lacto-ovo vegetarian and rice-based cuisines for males and females aged 9 years and older, excluding pregnant and lactating women.

All plans were set at energy levels for weight maintenance and aimed to include as many grain-based food servings as possible. The meal plans represented planning of a balanced diet for an individual (hence RDI values were used as a comparator) and a specifically created template was used to map out the food pattern in FoodWorks. Energy requirements were based on a BMI of $22.5 \mathrm{~kg} / \mathrm{m}^{2}$, light physical activity (physical activity level $=1 \cdot 6-1 \cdot 7$, as defined by FoodWorks) and the midpoint in years of each age group. BMI levels were determined using the average height and weight data for Australian males and females ${ }^{(18)}$ and the 50th centile for age for children ${ }^{(19)}$. Energy requirements were calculated in FoodWorks based on the nutrient reference value requirements for age. Meal plans were developed for each age group using:

1. all wholegrain foods (100\% WG);

2. half refined-grain and half wholegrain foods (50/50); and

3. all refined-grain foods ( $100 \% \mathrm{RG})$.

The 50/50 meal plans aimed to reach at least two servings each of refined grains and whole grains (i.e. $50 \%$ of four servings daily).

Wholegrain models were developed based on the recommended serving sizes stated in the $\mathrm{AGHE}^{(10)}$ and Food for Health: A Guide to Healthy Eating ${ }^{(7)}$. Refinedgrain foods were substituted in the 50/50 and 100\% RG meal plans; this substitution often required larger serving sizes to allow for energy equivalence between the plans. 
Food selection information for the two cuisines and recipes for each of the meal plans were obtained from literature searches, cuisine-based recipe books, consultation with experts for the cuisines and online recipe websites from magazines. For the rice-based meal plans where a food contained in a recipe was not available in the AUSNUT or AusFoods database, the online NUTTAB 2006 database $^{(20)}$ followed by the US Department of Agriculture's Nutrient Database for Standard Reference ${ }^{(21)}$ were used to obtain the nutrient information.

The reference values and nutrients for determining nutritional adequacy were as follows.

1. RDI: protein, thiamin, riboflavin, niacin equivalents, folate, retinol equivalents, vitamin $\mathrm{C}, \mathrm{Ca}, \mathrm{P}, \mathrm{Zn}, \mathrm{Fe}$ and $\mathrm{Mg}$.

2. AI: dietary fibre, $\mathrm{Na}$ and $\mathrm{K}$.

3. AMDR: total and saturated fat, carbohydrate and protein.

All meal plans were checked against the Food for Health (Dietary Guidelines) ${ }^{(7)}$ and $\mathrm{AGHE}^{(10)}$ food group recommendations. The food groups considered were breads/cereals, vegetables, fruit, dairy, legumes, eggs, nuts (including peanut butter) and extra foods. For the rice-based plans, meat was also considered in line with the legumes. Nutrient data were checked against upper limits for a given nutrient where available.

The average number of servings of grain-based foods daily was calculated over a 1 week $(7$ d) period (e.g. number of servings daily $=$ total number of servings divided by seven). Information on wholegrain food sources was obtained from the Food Standards Australia New Zealand website. The aim of the meal plans was to achieve the RDI and AI values through the inclusion of a variety of different foods and at least four grainbased servings. The ability to prepare the meals and the appropriateness in a food service setting were not considered when creating the meal plans.

\section{Results}

All lacto-ovo vegetarian meal plans were able to achieve the AI for fibre regardless of gender and age group. An example is shown in Table 1 demonstrating how the different levels of grain-based foods were equated within the breakfast meal. The nutrient values for the vegetarian plans are shown in Table 2. These nutrient values are an aggregate of all foods included in the meal plan multiplied by the frequency at which they were included.

Including four servings of grain-based foods for children aged 9-13 years limited total diet values for essential nutrients. For females, an average of 3.6 servings were included and for males $4 \cdot 0$ servings were achieved for most plans except $100 \%$ WG, where the average over 1 week was 3.9 servings/d. In these plans, increasing the grainbased food servings to four would have required reductions in other food groups with subsequent reductions in values for essential nutrients, particularly Fe. The lower energy requirements for young females equated to a stronger need for nutrient-dense food choices, as this age group of females needed to meet the same RDI for energy as the males $(1200 \mathrm{~kJ})$. These differences were adjusted for in the mid-meal snacks which served as key providers of essential nutrients, while the plans for males had more energy-dense foods such as low-fat ice cream and crackers with peanut butter to meet energy requirements.

Without the inclusion of meat in the meal plans, the greatest contributors to $\mathrm{Zn}$ were the dairy products cheese, milk and yoghurt. Incorporating $300 \mathrm{ml}$ of ageappropriate flavoured milk for children each day was an important contributor to the $\mathrm{Zn}$ levels in these meal plans.

All requirements for food groups were achieved. There were more than adequate servings of fruit and dairy food in the meal plans for the younger age group, although high levels of 'extra' foods, particularly for the males. The larger serving of low-fat ice cream was a major contributor. The energy value was not as high as other typical 'extra' foods, but the Ca level was not high enough to be considered as a serving of dairy food. Realistic children's food choices and preferences were considered; for example, an occasional (once weekly) serving of potato chips.

The male adolescents aged 14-18 years had the highest energy requirement of $\sim 12350 \mathrm{~kJ}$. To meet this requirement, the snacks between meals were again considered important for delivering additional nutrients. Extra grainbased food servings were added in the form of muffins and crackers available in wholegrain and refined-grain varieties. Cashew nuts were also included as a relatively good source of $\mathrm{Fe}$ in a vegetarian diet and a convenient snack.

The higher energy requirement for adults aged $\geq 19$ years, compared with younger children, meant meeting nutrient and food group requirements was not as challenging. Again, including grain-based foods within the snacks and additional servings as side dishes to main meals meant meeting the four servings of grain-based foods was feasible. In particular, for males, it was possible to boost the grainbased foods to an average of 5.7 servings/d with the inclusion of garlic bread, chapatti with curry and more grain-based snacks. With the lower energy requirements of females it was possible to meet, but not exceed four servings. Adult-appropriate drink choices were incorporated, in particular coffee and a glass of red wine twice weekly. Again, cashews and dried apricots were integral to providing adequate $\mathrm{Fe}$ in this vegetarian meal plan, with foods higher in complementary vitamin $\mathrm{C}$ added to increase bioavailability.

All rice-based meal plans were able to achieve the AI for fibre regardless of gender and age group. All plans also exceeded the AI for $\mathrm{Na}$ due to the inclusion of ingredients commonly used in Asian cooking. The meal plans and nutrient values of the rice-based diets are shown in Tables 3 and 4, respectively. 
Table 1 Meal plan for an entire day for a male adult consuming a lacto-ovo vegetarian diet, showing all wholegrain (line 1), $50 \%$ wholegrain-50\% refined grain (line 2 ) and all refined grain (line 3) for breakfast only. Remainder of meals show $100 \%$ wholegrain choices

\begin{tabular}{|c|c|c|c|c|c|c|}
\hline Day 1 & Day 2 & Day 3 & Day 4 & Day 5 & Day 6 & Day 7 \\
\hline \multicolumn{7}{|l|}{ Breakfast } \\
\hline $58 \mathrm{~g}$ cereal & $75 \mathrm{~g}$ cereal & $58 \mathrm{~g}$ cereal & 2 cereal biscuits & $0.5 \mathrm{C}$ untoasted muesli & $75 \mathrm{~g}$ cereal & 2 slices grain toast \\
\hline $\begin{array}{l}250 \mathrm{ml} \mathrm{skimmed} \\
(0 \cdot 15 \%) \text { milk }\end{array}$ & $\begin{array}{l}250 \mathrm{ml} \text { skimmed }(0 \cdot 15 \%) \\
\text { milk }\end{array}$ & $\begin{array}{l}250 \mathrm{ml} \text { skimmed }(0 \cdot 15 \%) \\
\text { milk }\end{array}$ & $\begin{array}{l}250 \mathrm{ml} \text { skimmed }(0 \cdot 15 \%) \\
\text { milk }\end{array}$ & $\begin{array}{l}250 \mathrm{ml} \text { skimmed }(0 \cdot 15 \%) \\
\text { milk }\end{array}$ & $\begin{array}{l}250 \mathrm{ml} \text { skimmed }(0 \cdot 15 \%) \\
\text { milk }\end{array}$ & 2 tsp margarine \\
\hline $\begin{array}{l}100 \mathrm{~g} \text { reduced-fat fruit } \\
\text { yoghurt }\end{array}$ & & $\begin{array}{l}100 \mathrm{~g} \text { reduced-fat fruit } \\
\text { yoghurt }\end{array}$ & $\begin{array}{l}100 \mathrm{~g} \text { reduced-fat fruit } \\
\text { yoghurt }\end{array}$ & $\begin{array}{l}100 \mathrm{~g} \text { reduced-fat fruit } \\
\text { yoghurt }\end{array}$ & & $\begin{array}{l}1 \text { poached egg, tomato, } \\
\text { mushrooms }\end{array}$ \\
\hline 2 slices grain toast & 2 slices grain toast & 2 slices grain toast & 2 slices grain toast & 2 slices grain toast & 2 slices grain toast & OR \\
\hline 2 tsp margarine & 2 tsp margarine & 2 tsp margarine & 2 tsp margarine & 2 tsp margarine & 2 tsp margarine & $\begin{array}{l}3 \text { wholemeal (whole- } \\
\text { wheat) pancakes, } \\
\text { berries, reduced-fat } \\
\text { ricotta, } 1 \text { tbsp honey }\end{array}$ \\
\hline \multirow[t]{2}{*}{$\begin{array}{l}1 \text { mug white coffee } \\
\text { with sweetener }\end{array}$} & $\begin{array}{l}1 \text { mug white coffee with } \\
\text { sweetener }\end{array}$ & $\begin{array}{l}1 \text { mug white coffee with } \\
\text { sweetener }\end{array}$ & $\begin{array}{l}1 \text { mug white coffee with } \\
\text { sweetener }\end{array}$ & $\begin{array}{l}1 \text { mug white coffee with } \\
\text { sweetener }\end{array}$ & $200 \mathrm{ml}$ vegetable juice & $200 \mathrm{ml}$ fruit juice \\
\hline & & & & & $\begin{array}{l}1 \text { mug white coffee with } \\
\text { sweetener }\end{array}$ & $\begin{array}{l}1 \text { mug white coffee with } \\
\text { sweetener }\end{array}$ \\
\hline OR & OR & OR & OR & OR & OR & OR \\
\hline $58 \mathrm{~g}$ cereal & $73 \mathrm{~g}$ cereal & $58 \mathrm{~g}$ cereal & 2 cereal biscuits & $1.5 \mathrm{C}$ cereal & $73 \mathrm{~g}$ cereal & $\begin{array}{l}3 \text { wholemeal (whole- } \\
\text { wheat) pancakes }\end{array}$ \\
\hline $\begin{array}{l}2 \text { slices grain toast } \\
\text { OR }\end{array}$ & $\begin{array}{l}2 \text { slices grain toast } \\
\text { OR }\end{array}$ & $\begin{array}{l}2 \text { slices grain toast } \\
\text { OR }\end{array}$ & $\begin{array}{l}2 \text { slices grain toast } \\
\text { OR }\end{array}$ & $\begin{array}{l}2 \text { slices grain toast } \\
\text { OR }\end{array}$ & $\begin{array}{l}2 \text { slices grain toast } \\
\text { OR }\end{array}$ & $\begin{array}{l}2 \text { slices grain toast } \\
\text { OR }\end{array}$ \\
\hline $1.7 \mathrm{C}$ cereal & $73 \mathrm{~g}$ cereal & $1.7 \mathrm{C}$ cereal & $28 \mathrm{~g}$ cereal & $1.5 \mathrm{C}$ cereal & $73 \mathrm{~g}$ cereal & 3.5 plain pancakes \\
\hline $\begin{array}{l}2 \text { thin slices white } \\
\text { toast }\end{array}$ & 2 thin slices white toast & 2 thin slices white toast & 2 thin slices white toast & 2 thin slices white toast & 2 thin slices white toast & 2 thin slices white toast \\
\hline \multicolumn{7}{|l|}{ Morning tea } \\
\hline 1 fruit & 1 fruit & 1 fruit & 1 fruit & 1 fruit & 1 fruit & 1 fruit \\
\hline 4 brown rice cakes & $200 \mathrm{~g}$ diet fruit yoghurt & 4 brown rice cakes & $200 \mathrm{~g}$ diet fruit yoghurt & & $200 \mathrm{~g}$ diet fruit yoghurt & $200 \mathrm{~g}$ diet fruit yoghurt \\
\hline 2 tbsp peanut butter & & 2 tbsp peanut butter & $\begin{array}{l}1 \text { wholemeal (whole-wheat) } \\
\text { fruit muffin }\end{array}$ & & 4 brown rice cakes & 2 oatmeal cookies \\
\hline $\begin{array}{l}1 \text { mug white coffee } \\
\text { with sweetener }\end{array}$ & $\begin{array}{l}1 \text { mug white coffee with } \\
\text { sweetener }\end{array}$ & $\begin{array}{l}1 \text { mug white coffee with } \\
\text { sweetener }\end{array}$ & $\begin{array}{l}1 \text { mug white coffee with } \\
\text { sweetener }\end{array}$ & $\begin{array}{l}1 \text { mug white coffee with } \\
\text { sweetener }\end{array}$ & $\begin{array}{l}2 \text { tbsp peanut butter } \\
1 \text { mug white coffee with } \\
\text { sweetener }\end{array}$ & $\begin{array}{l}1 \text { mug white coffee with } \\
\text { sweetener }\end{array}$ \\
\hline \multicolumn{7}{|l|}{ Lunch } \\
\hline Salad with cheese & $\begin{array}{l}2 \text { slices wholemeal } \\
\text { (whole-wheat) toast } \\
\text { and baked beans }\end{array}$ & $\begin{array}{l}\text { Cheese salad wholemeal } \\
\text { (whole-wheat) lavash }\end{array}$ & Salad and egg grain roll & $\begin{array}{l}\text { Wholemeal (whole-wheat) } \\
\text { pita bread with feta, } \\
\text { pumpkin and rocket }\end{array}$ & $\begin{array}{l}\text { Egg salad sandwich on } \\
2 \text { slices pumpernickel }\end{array}$ & $\begin{array}{l}\text { Vegetarian mini pizza } \\
\text { on grain English } \\
\text { muffin }\end{array}$ \\
\hline $\begin{array}{l}300 \mathrm{ml} \text { skimmed } \\
(0 \cdot 15 \%) \text { milk fruit } \\
\text { smoothie }\end{array}$ & $600 \mathrm{ml}$ water & $\begin{array}{l}300 \mathrm{ml} \text { skimmed }(0 \cdot 15 \%) \\
\text { milk fruit smoothie }\end{array}$ & $600 \mathrm{ml}$ water & $\begin{array}{l}300 \mathrm{ml} \text { skimmed }(0 \cdot 15 \%) \\
\text { milk fruit smoothie }\end{array}$ & $600 \mathrm{ml}$ water & $600 \mathrm{ml}$ water \\
\hline
\end{tabular}


Table 1 Continued

\begin{tabular}{|c|c|c|c|c|c|c|}
\hline Day 1 & Day 2 & Day 3 & Day 4 & Day 5 & Day 6 & Day 7 \\
\hline \multicolumn{7}{|l|}{ Afternoon tea } \\
\hline $0.25 \mathrm{C}$ nuts & $0.25 \mathrm{C}$ nuts & $0.25 \mathrm{C}$ nuts & $0.25 \mathrm{C}$ nuts & $0.25 \mathrm{C}$ nuts & $0.25 \mathrm{C}$ nuts & $0.25 \mathrm{C}$ nuts \\
\hline \multirow[t]{2}{*}{8 pieces dried fruit } & $\begin{array}{l}2 \text { wholemeal (whole-wheat) } \\
\text { English muffins }\end{array}$ & 8 pieces dried fruit & 8 pieces dried fruit & $\begin{array}{l}4 \text { grain crisp bread with } \\
\text { reduced-fat ricotta and } \\
\text { tomato }\end{array}$ & & 8 pieces dried fruit \\
\hline & $\begin{array}{l}2 \text { tsp diet jam } \\
2 \text { tsp margarine }\end{array}$ & & 1 fruit & & 1 fruit & 1 fruit \\
\hline $\begin{array}{l}1 \text { mug white coffee } \\
\text { with sweetener }\end{array}$ & $\begin{array}{l}1 \text { mug white coffee with } \\
\text { sweetener }\end{array}$ & $\begin{array}{l}1 \text { mug white coffee with } \\
\text { sweetener }\end{array}$ & $\begin{array}{l}1 \text { mug white coffee with } \\
\text { sweetener }\end{array}$ & $\begin{array}{l}1 \text { mug white coffee with } \\
\text { sweetener }\end{array}$ & $\begin{array}{l}1 \text { mug white coffee with } \\
\text { sweetener }\end{array}$ & $\begin{array}{l}1 \text { mug white coffee with } \\
\text { sweetener }\end{array}$ \\
\hline \multicolumn{7}{|l|}{ Dinner } \\
\hline $\begin{array}{l}0.5 \mathrm{C} \text { wholemeal } \\
\text { (whole-wheat) } \\
\text { pasta }\end{array}$ & $0.75 \mathrm{C}$ vegetable curry & $\begin{array}{l}290 \mathrm{~g} \text { wholemeal } \\
\text { (whole-wheat) vegetable } \\
\text { lasagne }\end{array}$ & $\begin{array}{l}1.5 \mathrm{C} \text { satay tofu and } \\
\text { vegetables }\end{array}$ & $\begin{array}{l}1 \mathrm{C} \text { wholemeal } \\
\text { (whole-wheat) pasta } \\
\text { with pesto }\end{array}$ & $\begin{array}{l}1 \mathrm{C} \text { vegetable and grain } \\
\text { risotto }\end{array}$ & $\begin{array}{l}300 \mathrm{~g} \text { Moroccan spiced } \\
\text { vegetable and grain } \\
\text { salad }\end{array}$ \\
\hline $\begin{array}{l}0.75 \mathrm{C} \text { tomato-based } \\
\text { pasta sauce }\end{array}$ & $1 \mathrm{C}$ wild rice & 1 grain roll with garlic butter & $1 \mathrm{C}$ brown rice & $300 \mathrm{~g}$ mixed vegetables & & \\
\hline $30 \mathrm{~g}$ cheese & $\begin{array}{l}2 \text { slices wholemeal } \\
\text { (whole-wheat) bread }\end{array}$ & $30 \mathrm{~g}$ reduced-fat cheese & & & & \\
\hline $\begin{array}{l}300 \mathrm{~g} \text { mixed } \\
\text { vegetables }\end{array}$ & $50 \mathrm{~g}$ yoghurt dip & $300 \mathrm{~g}$ mixed vegetables & & & & \\
\hline $200 \mathrm{ml}$ soft drink & $200 \mathrm{ml}$ soft drink & $200 \mathrm{ml}$ soft drink & $200 \mathrm{ml}$ soft drink & $200 \mathrm{ml}$ soft drink & 1 glass wine & 1 glass wine \\
\hline $600 \mathrm{ml}$ water & $600 \mathrm{ml}$ water & $600 \mathrm{ml}$ water & $600 \mathrm{ml}$ water & $600 \mathrm{ml}$ water & $600 \mathrm{ml}$ water & $600 \mathrm{ml}$ water \\
\hline \multirow[t]{2}{*}{$\begin{array}{l}\text { Supper } \\
2 \mathrm{C} \text { popcorn }\end{array}$} & 1 fruit & $2 \mathrm{C}$ fruit salad & 1 fruit & $200 \mathrm{~g}$ low-fat ice cream & $200 \mathrm{~g}$ low-fat ice cream & $\begin{array}{l}12 \text { wholegrain rice } \\
\text { crackers }\end{array}$ \\
\hline & $\begin{array}{l}0.5 \mathrm{C} \text { reduced-fat custard } \\
125 \mathrm{ml} \text { vegetable juice }\end{array}$ & $\begin{array}{l}1 \mathrm{C} \text { reduced-fat custard } \\
125 \mathrm{ml} \text { vegetable juice }\end{array}$ & $\begin{array}{l}0.5 \mathrm{C} \text { reduced-fat custard } \\
125 \mathrm{ml} \text { vegetable juice }\end{array}$ & $\begin{array}{l}200 \mathrm{~g} \text { fruit crumble } \\
125 \mathrm{ml} \text { vegetable juice }\end{array}$ & $\begin{array}{l}200 \mathrm{~g} \text { fruit crumble } \\
125 \mathrm{ml} \text { vegetable juice }\end{array}$ & $\begin{array}{l}2 \mathrm{tbsp} \text { salsa } \\
125 \mathrm{ml} \text { vegetable juice }\end{array}$ \\
\hline
\end{tabular}

C, cup; tbsp, tablespoon; tsp, teaspoon.

Grain breads, rolls and toasts contain intact grains, while wholemeal (whole-wheat) breads, rolls and toasts contain partially ground grains.

Each food group was represented by individual foods providing total for $7 \mathrm{~d}$ options. 
Table 2 Daily nutrient values for lacto-ovo vegetarian meal plans, mean and percentage of the Recommended Dietary Intake (\%RDI)

\begin{tabular}{|c|c|c|c|c|c|c|c|c|c|c|c|c|c|c|c|c|c|c|}
\hline \multirow[b]{3}{*}{ Nutrient } & \multicolumn{6}{|c|}{$9-13$ years } & \multicolumn{6}{|c|}{$14-18$ years } & \multicolumn{6}{|c|}{$\geq 19$ years } \\
\hline & \multicolumn{2}{|c|}{$100 \%$ WG } & \multicolumn{2}{|c|}{$50 / 50$} & \multicolumn{2}{|c|}{$100 \% R G$} & \multicolumn{2}{|c|}{$100 \%$ WG } & \multicolumn{2}{|c|}{$50 / 50$} & \multicolumn{2}{|c|}{$100 \%$ RG } & \multicolumn{2}{|c|}{$100 \%$ WG } & \multicolumn{2}{|c|}{$50 / 50$} & \multicolumn{2}{|c|}{$100 \% R G$} \\
\hline & $\mathrm{F}$ & $\mathrm{M}$ & $\mathrm{F}$ & $\mathrm{M}$ & $\mathrm{F}$ & $M$ & $\mathrm{~F}$ & $M$ & $\mathrm{~F}$ & $M$ & $\mathrm{~F}$ & $\mathrm{M}$ & $\mathrm{F}$ & $\mathrm{M}$ & $\mathrm{F}$ & $M$ & $\mathrm{~F}$ & M \\
\hline Energy (kJ) & 7996 & 9212 & 7996 & 9212 & 7995 & 9211 & 9475 & 12357 & 9474 & 12356 & 9473 & 12358 & 8816 & 12086 & 8816 & 12086 & 8817 & 12088 \\
\hline $\mathrm{CHO}(\mathrm{g})$ & 290 & 327 & 294 & 329 & 299 & 333 & 322 & 389 & 323 & 390 & 327 & 402 & 280 & 372 & 281 & 373 & 285 & 3085 \\
\hline Protein (g) & 78 & 91 & 77 & 89 & 77 & 89 & 88 & 112 & 87 & 111 & 87 & 109 & 81 & 108 & 79 & 106 & 80 & 105 \\
\hline Total fat $(\mathrm{g})$ & 42 & 52 & 41 & 53 & 41 & 52 & 61 & 96 & 63 & 97 & 62 & 94 & 61 & 95 & 63 & 96 & 62 & 93 \\
\hline SFA (g) & 14 & 14 & 14 & 15 & 14 & 15 & 17 & 26 & 18 & 27 & 18 & 26 & 15 & 25 & 16 & 26 & 16 & 26 \\
\hline PUFA (g) & 8 & 12 & 8 & 12 & 7 & 12 & 12 & 20 & 11 & 20 & 11 & 18 & 12 & 20 & 12 & 20 & 12 & 19 \\
\hline MUFA (g) & 16 & 20 & 16 & 21 & 15 & 20 & 28 & 43 & 28 & 43 & 28 & 42 & 29 & 43 & 29 & 43 & 29 & 42 \\
\hline $\mathrm{CHO}(\% \mathrm{E})$ & 63 & 62 & 64 & 62 & 64 & 62 & 59 & 55 & 59 & 55 & 59 & 56 & 56 & 53 & 56 & 53 & 56 & 55 \\
\hline Protein (\%E) & 17 & 17 & 17 & 17 & 17 & 17 & 16 & 16 & 16 & 15 & 16 & 15 & 16 & 16 & 16 & 15 & 16 & 15 \\
\hline Fat $(\% \mathrm{E})$ & 20 & 21 & 20 & 22 & 19 & 21 & 24 & 29 & 25 & 30 & 25 & 29 & 27 & 30 & 27 & 30 & 27 & 29 \\
\hline SFA (\%E) & 6 & 5 & 6 & 6 & 6 & 6 & 6 & 8 & 7 & 8 & 7 & 8 & 6 & 7 & 7 & 8 & 7 & 8 \\
\hline PUFA (\%E) & 4 & 5 & 4 & 5 & 3 & 5 & 5 & 6 & 4 & 6 & 4 & 5 & 5 & 6 & 5 & 6 & 5 & 6 \\
\hline MUFA (\%E) & 7 & 8 & 7 & 8 & 7 & 8 & 11 & 13 & 11 & 13 & 11 & 12 & 12 & 13 & 12 & 13 & 12 & 13 \\
\hline SFA (\% fat) & 37 & 31 & 37 & 32 & 37 & 32 & 30 & 29 & 31 & 30 & 31 & 30 & 27 & 29 & 28 & 30 & 29 & 30 \\
\hline PUFA (\% fat) & 21 & 26 & 21 & 25 & 21 & 25 & 21 & 22 & 20 & 22 & 20 & 21 & 22 & 23 & 21 & 22 & 21 & 49 \\
\hline MUFA (\% fat) & 42 & 43 & 42 & 43 & 42 & 43 & 49 & 48 & 49 & 48 & 49 & 49 & 51 & 48 & 51 & 48 & 50 & 49 \\
\hline Alcohol (g) & 0 & 0 & 0 & 0 & 0 & 0 & 0 & 0 & 0 & 0 & 0 & 0 & 4 & 4 & 4 & 4 & 4 & 4 \\
\hline Alcohol (\%E) & 0 & 0 & 0 & 0 & 0 & 0 & 0 & 0 & 0 & 0 & 0 & 0 & 1 & 1 & 1 & 1 & 1 & 1 \\
\hline P:S & 0.57 & 0.86 & 0.57 & 0.93 & 0.50 & 0.93 & 0.71 & 0.77 & 0.60 & 0.74 & $0 \cdot 60$ & 0.69 & $0 \cdot 81$ & 0.81 & 0.75 & $0 \cdot 80$ & 0.75 & 0.73 \\
\hline Thiamin (mg) & & & & & & & & & & & & & & & & & & \\
\hline Mean & $1 \cdot 7$ & $1 \cdot 8$ & 1.9 & $2 \cdot 0$ & $1 \cdot 8$ & $2 \cdot 0$ & $2 \cdot 3$ & $2 \cdot 4$ & 2.5 & 2.5 & 2.5 & $2 \cdot 4$ & $2 \cdot 4$ & $2 \cdot 4$ & 2.5 & $2 \cdot 5$ & $2 \cdot 5$ & $2 \cdot 4$ \\
\hline \%RDI & 243 & 257 & 271 & 286 & 257 & 286 & 256 & 240 & 278 & 250 & 278 & 240 & 267 & 240 & 278 & 250 & 278 & 240 \\
\hline Riboflavin (mg) & & & & & & & & & & & & & & & & & & \\
\hline Mean & $3 \cdot 1$ & $3 \cdot 2$ & 3.4 & 3.5 & $3 \cdot 3$ & 3.5 & 3.8 & $3 \cdot 7$ & $4 \cdot 1$ & $4 \cdot 0$ & $4 \cdot 0$ & $4 \cdot 0$ & $3 \cdot 3$ & $3 \cdot 4$ & $3 \cdot 6$ & $3 \cdot 7$ & 3.6 & $3 \cdot 7$ \\
\hline \%RDI & 388 & 400 & 425 & 438 & 413 & 438 & 422 & 336 & 456 & 364 & 444 & 364 & 367 & 309 & 400 & 336 & 400 & 336 \\
\hline Niacin equiv. ( & & & & & & & & & & & & & & & & & & \\
\hline Mean & 35 & 42 & 35 & 41 & 34 & 39 & 43 & 52 & 42 & 51 & 40 & 48 & 43 & 54 & 41 & 52 & 40 & 50 \\
\hline \%RDI & 388 & 467 & 388 & 456 & 378 & 433 & 391 & 433 & 381 & 425 & 363 & 400 & 391 & 450 & 372 & 433 & 363 & 417 \\
\hline Vitamin C (mg) & & & & & & & & & & & & & & & & & & \\
\hline Mean & 281 & 281 & 281 & 281 & 286 & 286 & 225 & 284 & 225 & 284 & 231 & 289 & 203 & 265 & 203 & 265 & 209 & 271 \\
\hline \%RDI & 1004 & 1004 & 1004 & 1004 & 1021 & 1021 & 804 & 1014 & 804 & 1014 & 821 & 1032 & 677 & 883 & 677 & 883 & 697 & 900 \\
\hline Total folate $(\mu \mathrm{g}$ & & & & & & & & & & & & & & & & & & \\
\hline Mean & 388 & 419 & 378 & 399 & 427 & 451 & 364 & 482 & 344 & 462 & 425 & 503 & 361 & 448 & 341 & 428 & 422 & 470 \\
\hline \%RDI & 155 & 168 & 151 & 160 & 171 & 180 & 110 & 138 & 104 & 140 & 129 & 152 & 113 & 140 & 107 & 134 & 132 & 147 \\
\hline Total retinol eq & & & & & & & & & & & & & & & & & & \\
\hline Mean & $2287 \dagger$ & $2258 t$ & $2288+$ & $2260 t$ & $2474 t$ & $2448+$ & 1501 & 2573 & 1503 & 2574 & 1691 & 2762 & $3210+$ & 2059 & $3211+$ & 2060 & $3399+$ & 2248 \\
\hline \%RDI & 545 & 507 & 545 & 508 & 589 & 550 & 309 & 408 & 310 & 409 & 349 & 438 & 642 & 329 & 642 & 330 & 680 & 360 \\
\hline $\mathrm{Na}(\mathrm{mg})^{\star}$ & $2454 t$ & $2543 t$ & $2496+$ & $2751 t$ & $2630+$ & $2890 t$ & $2602 t$ & $3164 t$ & $2819+$ & $3368+$ & $2972+$ & $3222 \dagger$ & $2511 t$ & $3389 t$ & $2716+$ & $3597 t$ & $2870+$ & $3450 t$ \\
\hline K (mg) & & & & & & & & & & & & & & & & & & \\
\hline Mean & 4827 & 5106 & 4695 & 4943 & 4571 & 4820 & 5254 & 6231 & 5090 & 6066 & 4960 & 5877 & 5097 & 6329 & 4939 & 6164 & 4809 & 5975 \\
\hline \%RDI & 193 & 170 & 198 & 165 & 183 & 161 & 202 & 173 & 196 & 169 & 191 & 163 & 182 & 167 & 176 & 162 & 172 & 157 \\
\hline$M g(\mathrm{mg})$ & & & & & & & & & & & & & & & & & & \\
\hline Mean & 515 & 585 & 461 & 511 & 423 & 473 & 654 & 784 & 580 & 710 & 542 & 639 & 643 & 801 & 571 & 726 & 532 & 656 \\
\hline \%RDI & 258 & 293 & 231 & 256 & 212 & 237 & 354 & 231 & 193 & 209 & 181 & 188 & 243 & 243 & 215 & 220 & 201 & 199 \\
\hline $\mathrm{Ca}(\mathrm{mg})$ & & & & & & & & & & & & & & & & & & \\
\hline Mean & 1742 & 1870 & 1733 & 1865 & 1749 & 1880 & 1776 & 2120 & 1772 & 2115 & 1787 & 2092 & 1495 & 1939 & 1493 & 1934 & 1509 & 1911 \\
\hline \%RDI & 218 & 234 & 217 & 233 & 218 & 235 & 169 & 202 & 169 & 201 & 170 & 199 & 178 & 231 & 177 & 230 & 180 & 228 \\
\hline $\mathrm{P}(\mathrm{mg})$ & & & & & & & & & & & & & & & & & & \\
\hline Mean & 1919 & 2184 & 1770 & 1992 & 1698 & 1920 & 2192 & 2669 & 2000 & 2478 & 1929 & 2353 & 1988 & 2547 & 1803 & 2355 & 1732 & 2231 \\
\hline \%RDI & 182 & 207 & 168 & 189 & 161 & 182 & 208 & 253 & 190 & 235 & 183 & 223 & 343 & 439 & 311 & 406 & 299 & 385 \\
\hline
\end{tabular}


The meal plans for children aged 9-13 years were not able to incorporate the total four servings of grain-based foods and still meet the RDI for all nutrients within the appropriate energy requirement. Initial meal plans that did include four servings each day were not able to meet the RDI for Fe and folate. The grain servings were mostly within main meals while mid-meal snacks contained nutrient-rich foods to meet the food group targets for growing children, predominantly fruit and dairy.

Meeting dairy requirements was also a challenge in the rice-based (quasi-Asian) diets across all age groups. For children only 1.8 dairy servings/d, not the recommended two servings were achieved, although the plans did reach the lower end of the RDI range for $\mathrm{Ca}$. Meeting dairy requirements was complicated as breakfast cereal and milk are not typical foods, and a very high prevalence of lactose intolerance exists among the Asian population ${ }^{(22)}$. Fortified soya beverages were used as daily drinks to contribute to 'dairy' and $\mathrm{Ca}$.

Increased energy requirements for adolescents aged 14-18 years made it feasible to include four servings of grain foods in 100\% RG and 100\% WG meal plans. This was met with larger serving sizes of grain foods within meals and occasional grain-based snacks such as cookies and pancakes. While many of the RDI values are similar, these needed to be met with less energy for females than for males. More nutrient-dense foods were included to account for these differences. For example, for females, morning tea fruit selections included grapefruit, banana and mango which provided $64 \mu \mathrm{g}$ (16\% RDI) of folate; in comparison with the male fruit choices of plums, kiwifruit and lychees which provided $\sim 20 \%$ of this amount of folate. Fruit was a major contributor to energy and essential micronutrients in the plans and the inclusion of fresh tropical fruits, juice and dried fruit contributed to an average of four servings daily.

It was again a challenge to meet the four servings of grain-based foods for adults aged $\geq 19$ years because of the lack of grain-based snacks in the Asian cuisine. Again, grain-based foods were confined predominantly to the main meals and the increased serving size of these meals compared with children was the best strategy for increasing the total number of grain-based foods servings daily. Again, fruit contributed many of the snacks in these plans and specifically berries were included to boost the micronutrient content of the female diets.

\section{Discussion}

Achievement of the RDI and AI requirements for the lacto-Ovo vegetarian and quasi-Asian meal plans required careful consideration of food choices depending on the target age group and gender. $\mathrm{Zn}, \mathrm{Fe}$, folate and $\mathrm{Na}$ levels provided the most challenge. The first three were often below the nutrient reference value while the latter was generally above the value. $\mathrm{Zn}, \mathrm{Fe}$ and folate were 
Table 3 Meal plan for an entire day for a female adult consuming a rice-based (quasi-Asian) diet, showing all wholegrain (line 1), $50 \%$ wholegrain-50\% refined (line 2 ) and all refined grain (line 3) for breakfast only. Remainder of meals show $100 \%$ wholegrain choices

\begin{tabular}{|c|c|c|c|c|c|c|}
\hline Day 1 & Day 2 & Day 3 & Day 4 & Day 5 & Day 6 & Day 7 \\
\hline \multicolumn{7}{|l|}{ Breakfast } \\
\hline \multirow[t]{2}{*}{$\begin{array}{l}\text { Wholegrain nasi lemak, } \\
\text { egg, peanut, } \\
\text { cucumber, dried fish } \\
\text { sambal }\end{array}$} & $\begin{array}{l}2 \text { slices grain toast, kaya } \\
\text { paste }\end{array}$ & Kao tom & Beef pho & $\begin{array}{l}\text { Grain roll, fried egg and } \\
\text { soya sauce }\end{array}$ & $\begin{array}{l}\text { Wholegrain congee, } \\
\text { poached egg, soya } \\
\text { sauce }\end{array}$ & Wholegrain ensaymada \\
\hline & & $1 \mathrm{C}$ brown rice & $250 \mathrm{~g}$ soba noodles & & & \\
\hline $\begin{array}{l}200 \mathrm{ml} \text { tea } \\
\text { OR }\end{array}$ & $\begin{array}{l}200 \mathrm{ml} \text { tea } \\
\text { OR }\end{array}$ & $\begin{array}{l}200 \mathrm{ml} \text { tea } \\
\text { OR }\end{array}$ & $\begin{array}{l}200 \mathrm{ml} \text { tea } \\
\text { OR }\end{array}$ & $\begin{array}{l}200 \mathrm{ml} \text { tea } \\
\text { OR }\end{array}$ & $\begin{array}{l}200 \mathrm{ml} \text { tea } \\
\text { OR }\end{array}$ & $\begin{array}{l}200 \mathrm{ml} \text { tea } \\
\text { OR }\end{array}$ \\
\hline Wholegrain nasi lemak & 2 slices grain toast & $1 \cdot 16 \mathrm{C}$ white rice & $250 \mathrm{~g}$ rice noodles & $\begin{array}{l}25 \mathrm{~g} \text { Vietnamese bread } \\
\text { stick }\end{array}$ & White rice congee & White rice ensaymada \\
\hline OR & OR & OR & OR & OR & OR & OR \\
\hline Malay nasi lemak & 2 thin slices white toast & $1 \cdot 16 \mathrm{C}$ white rice & $250 \mathrm{~g}$ rice noodles & $\begin{array}{l}25 \mathrm{~g} \text { Vietnamese bread } \\
\text { stick }\end{array}$ & White rice congee & White rice ensaymada \\
\hline \multicolumn{7}{|l|}{ Morning tea } \\
\hline $\begin{array}{l}200 \mathrm{~g} \text { reduced-fat plain } \\
\text { yoghurt }\end{array}$ & $\begin{array}{l}250 \mathrm{ml} \text { fortified soya } \\
\text { beverage }\end{array}$ & $\begin{array}{l}200 \mathrm{~g} \text { reduced-fat plain } \\
\text { yoghurt }\end{array}$ & $\begin{array}{l}200 \mathrm{~g} \text { reduced-fat plain } \\
\text { yoghurt }\end{array}$ & $\begin{array}{l}250 \mathrm{ml} \text { fortified soya } \\
\text { beverage }\end{array}$ & $\begin{array}{l}200 \mathrm{~g} \text { reduced-fat plain } \\
\text { yoghurt }\end{array}$ & $\begin{array}{l}250 \mathrm{ml} \text { fortified soya } \\
\text { beverage }\end{array}$ \\
\hline $\begin{array}{l}0.5 \mathrm{C} \text { sliced fruit } \\
0.25 \mathrm{C} \text { mixed nuts, fruit } \\
\text { and seeds }\end{array}$ & 1 fruit & $1 \mathrm{C}$ sliced fruit & $\begin{array}{l}0.5 \mathrm{C} \text { sliced fruit } \\
0.25 \mathrm{C} \text { mixed nuts, fruit and } \\
\text { seeds }\end{array}$ & 1 fruit & $0.5 \mathrm{C}$ sliced fruit & $0.5 \mathrm{C}$ sliced fruit \\
\hline $200 \mathrm{ml}$ water & $125 \mathrm{ml}$ fruit juice & $200 \mathrm{ml}$ water & $125 \mathrm{ml}$ fruit juice & $200 \mathrm{ml}$ water & $125 \mathrm{ml}$ fruit juice & $200 \mathrm{ml}$ water \\
\hline \multicolumn{7}{|l|}{ Lunch } \\
\hline $\begin{array}{l}8 \text { pieces brown rice } \\
\text { sushi, soya sauce }\end{array}$ & Vietnamese rice paper rolls & 2 wholegrain jaioz & $\begin{array}{l}2 \text { wholemeal (whole-wheat) } \\
\text { fish cakes }\end{array}$ & Asian vegetable soup & 2 slices grain toast & $1 \mathrm{C}$ wholegrain lugaw \\
\hline $\begin{array}{l}\text { Steamed vegetables } \\
\text { and tuna }\end{array}$ & $\begin{array}{l}2 \mathrm{C} \text { salad vegetables, soya } \\
\text { sauce }\end{array}$ & 2 fish balls, chilli sauce & $\begin{array}{l}1.5 \mathrm{C} \text { steamed vegetables, } \\
\text { chilli sauce }\end{array}$ & $250 \mathrm{~g}$ soba noodles & $0.75 \mathrm{C}$ soya beans & $\begin{array}{l}1.5 \mathrm{C} \text { steamed Asian } \\
\text { greens }\end{array}$ \\
\hline $\begin{array}{l}\text { Seafood soup with } \\
\text { seaweed }\end{array}$ & & Poached egg & $1 \mathrm{C}$ brown rice & 1 chicken kebab & & \\
\hline 3 rice cakes with rye & & $\begin{array}{l}0.5 \mathrm{C} \text { salad vegetables, } \\
\text { soya sauce } \\
0.5 \mathrm{C} \text { brown rice }\end{array}$ & & 3 rice cakes with rye & & \\
\hline $200 \mathrm{ml}$ tea & $200 \mathrm{ml}$ tea & $200 \mathrm{ml}$ tea & $200 \mathrm{ml}$ tea & $200 \mathrm{ml}$ tea & $200 \mathrm{ml}$ tea & $200 \mathrm{ml}$ tea \\
\hline \multicolumn{7}{|l|}{ Afternoon tea } \\
\hline $\begin{array}{l}0.5 \mathrm{C} \text { Asian noodle, } \\
\text { peanut and soya mix }\end{array}$ & $1 \mathrm{C}$ mixed fruit & $\begin{array}{l}14 \text { brown rice crackers } \\
\text { with fish spread and } \\
\text { miso soup }\end{array}$ & $\begin{array}{l}200 \mathrm{~g} \text { reduced-fat fruit } \\
\text { yoghurt }\end{array}$ & $1 \mathrm{C}$ mixed fruit & $\begin{array}{l}200 \mathrm{~g} \text { reduced fat fruit } \\
\text { yoghurt }\end{array}$ & $0.25 \mathrm{C}$ peanuts \\
\hline $\begin{array}{l}250 \mathrm{ml} \text { fortified soya } \\
\text { beverage }\end{array}$ & $200 \mathrm{ml}$ water & $\begin{array}{l}250 \mathrm{ml} \text { fortified soya } \\
\text { beverage }\end{array}$ & $200 \mathrm{ml}$ water & $\begin{array}{l}250 \mathrm{ml} \text { fortified soya } \\
\text { beverage }\end{array}$ & $\begin{array}{l}250 \mathrm{ml} \text { fortified soya } \\
\text { beverage }\end{array}$ & $200 \mathrm{ml}$ water \\
\hline \multicolumn{7}{|l|}{ Dinner } \\
\hline $\begin{array}{l}0.75 \mathrm{C} \text { beef in black } \\
\text { bean sauce }\end{array}$ & $\begin{array}{l}1 \text { C miso with tofu, } \\
\text { seaweed }\end{array}$ & 1 fish fillet steamed & Miso-glazed salmon & $\begin{array}{l}\text { Ginger chicken with } \\
\text { Asian greens }\end{array}$ & Char kway teow & Dol sot bibimbap \\
\hline $\begin{array}{l}1.5 \mathrm{C} \text { steamed } \\
\text { vegetables }\end{array}$ & $\begin{array}{l}1.5 \mathrm{C} \text { Asian steamed } \\
\text { greens } \\
1 \mathrm{C} \text { green salad with } \\
\text { dressing }\end{array}$ & $\begin{array}{l}150 \mathrm{~g} \text { kimchi, soya } \\
\text { sauce }\end{array}$ & $2 \mathrm{C}$ steamed vegetables & & $\begin{array}{l}1.5 \mathrm{C} \text { steamed Asian } \\
\text { vegetables }\end{array}$ & \\
\hline
\end{tabular}


achieved in the majority of plans through required food choices seen as rich sources of each nutrient. This was of greater concern in the vegetarian meal plans where the avoidance of meat or fish reduced the range of choices. In these plans the consideration of non-haem $\mathrm{Fe}$ and its interactions with vitamin $\mathrm{C}$ was also addressed to ensure the bioavailability was maximised.

For the $100 \%$ WG meal plans, these nutrients were not as difficult to achieve due to the inherent nutrient density of the grains. Breakfast maximised the use of fortified cereal products helping to increase target nutrient levels, especially when compared with a bread/toast-based breakfast. The $\mathrm{Fe}$ content of the rice-based plans for $100 \%$ WG and 50/50 plans could have further been increased if black rice ${ }^{(23)}$ was used rather than the more readily available brown rice. However, the plans were based on Westernised cuisine and food composition databases. While each of the 100\% WG and some 50/50 plans did include brown rice, the majority of Asian cuisines are based on a number of different varieties of white rice only. While in a meal planning and cooking sense it was possible to substitute white rice for brown, whether this would be an acceptable substitution to the Asian population remains to be seen.

$\mathrm{Na}$ levels were above the AI in most meal plans. In the vegetarian plans this was primarily attributed to the high $\mathrm{Na}$ content of processed and packaged foods (not usually grain-based), which were included to reflect common Western eating patterns and a need for convenience. While grain-based foods did contribute $\mathrm{Na}$ to the meal plans, it was the cumulative effect of foods such as pre-packaged pasta and simmer sauces, deli meats, pre-packaged mixed spices and vegetable stock that resulted in the overall high $\mathrm{Na}$ load. The $\mathrm{Na}$ content of all of the rice-based plans exceeded the AI, due to the inclusion of processed and packaged foods (not usually grain-based) and Na-rich ingredients such as soya, fish and oyster sauce, stock, miso and seafood ingredients such as shrimp paste, seaweed and preserved fish. Inclusion of these foods was necessary to accurately reflect modern Asian cuisine. Grain-based foods were again not a major contributor to the Na load. Thus, the whole of diet was responsible for the excessive $\mathrm{Na}$ load, which is likely to be an accurate reflection of $\mathrm{Na}$ intake within the context of traditional Asian cuisine.

It is notable that the composition of all vegetarian and rice-based meal plans was sufficient to meet the AI for fibre. The primary difference between the vegetarian grain-based food meal plans was the 100\% RG plans having an average of $30 \%$ less fibre than the $100 \% \mathrm{WG}$. The difference in fibre content was marginal for the $100 \%$ RG and 100\% WG rice-based meal plans, as a cup of brown rice has only one-third of the fibre $(1.5 \mathrm{~g} / 100 \mathrm{~g})$ of wholemeal (whole-wheat) pasta $(5 \cdot 7 \mathrm{~g} / 100 \mathrm{~g})$ and $20 \%$ less fibre than two slices of grain bread (containing intact grains; $4 \cdot 1 \mathrm{~g} / 100 \mathrm{~g}$ ) or two Weetbix (whole-wheat cereal; $3 \cdot 2 \mathrm{~g} / 100 \mathrm{~g}$ ). Hence, overall the fibre content was 
Table 4 Daily nutrient values for rice-based (quasi-Asian) meal plans, mean and percentage of the Recommended Dietary Intake (\%RDI)

\begin{tabular}{|c|c|c|c|c|c|c|c|c|c|c|c|c|c|c|c|c|c|c|}
\hline \multirow[b]{3}{*}{ Nutrient } & \multicolumn{6}{|c|}{$9-13$ years } & \multicolumn{6}{|c|}{$14-18$ years } & \multicolumn{6}{|c|}{$\geq 19$ years } \\
\hline & \multicolumn{2}{|c|}{$100 \%$ WG } & \multicolumn{2}{|c|}{$50 / 50$} & \multicolumn{2}{|c|}{$100 \% R G$} & \multicolumn{2}{|c|}{$100 \%$ WG } & \multicolumn{2}{|c|}{$50 / 50$} & \multicolumn{2}{|c|}{$100 \% R G$} & \multicolumn{2}{|c|}{$100 \%$ WG } & \multicolumn{2}{|c|}{$50 / 50$} & \multicolumn{2}{|c|}{$100 \%$ RG } \\
\hline & $\mathrm{F}$ & M & $\mathrm{F}$ & M & $\mathrm{F}$ & $M$ & $\mathrm{~F}$ & $M$ & $\mathrm{~F}$ & M & $\mathrm{F}$ & M & $\mathrm{F}$ & $M$ & $\mathrm{~F}$ & M & $\mathrm{F}$ & M \\
\hline Energy (kJ) & 7995 & 9212 & 7996 & 9212 & 7996 & 9212 & 9475 & 12354 & 9475 & 12357 & 9475 & 12355 & 8817 & 12084 & 8817 & 12085 & 8815 & 12086 \\
\hline $\mathrm{CHO}(\mathrm{g})$ & 232 & 283 & 237 & 287 & 240 & 290 & 279 & 340 & 282 & 342 & 284 & 345 & 259 & 319 & 261 & 323 & 264 & 327 \\
\hline Protein (g) & 101 & 107 & 98 & 104 & 94 & 101 & 116 & 146 & 114 & 147 & 110 & 143 & 111 & 149 & 108 & 145 & 105 & 141 \\
\hline Total fat (g) & 60 & 67 & 59 & 66 & 58 & 65 & 71 & 104 & 70 & 105 & 70 & 104 & 65 & 104 & 64 & 103 & 64 & 102 \\
\hline SFA (g) & 15 & 17 & 15 & 17 & 15 & 16 & 17 & 25 & 17 & 26 & 17 & 26 & 16 & 26 & 16 & 25 & 16 & 25 \\
\hline PUFA (g) & 19 & 20 & 18 & 19 & 17 & 19 & 23 & 30 & 23 & 29 & 22 & 28 & 19 & 29 & 19 & 28 & 18 & 28 \\
\hline MUFA (g) & 20 & 23 & 20 & 22 & 19 & 22 & 23 & 38 & 23 & 38 & 23 & 38 & 22 & 39 & 22 & 38 & 22 & 38 \\
\hline $\mathrm{CHO}(\% \mathrm{E})$ & 48 & 53 & 51 & 51 & 52 & 54 & 50 & 48 & 51 & 48 & 52 & 48 & 51 & 45 & 51 & 46 & 52 & 47 \\
\hline Protein (\%E) & 22 & 20 & 21 & 19 & 20 & 19 & 21 & 20 & 20 & 20 & 20 & 20 & 22 & 21 & 21 & 21 & 20 & 20 \\
\hline Fat $(\% \mathrm{E})$ & 29 & 27 & 27 & 27 & 27 & 27 & 28 & 32 & 27 & 32 & 27 & 32 & 28 & 32 & 27 & 32 & 27 & 32 \\
\hline SFA (\% fat) & 28 & 28 & 28 & 28 & 28 & 29 & 27 & 27 & 27 & 26 & 27 & 28 & 28 & 25 & 28 & 24 & 29 & 25 \\
\hline PUFA (\% fat) & 35 & 34 & 34 & 33 & 34 & 33 & 37 & 32 & 36 & 29 & 36 & 31 & 34 & 28 & 34 & 27 & 33 & 28 \\
\hline MUFA (\% fat) & 37 & 38 & 37 & 38 & 38 & 38 & 37 & 41 & 37 & 38 & 37 & 41 & 38 & 38 & 38 & 37 & 39 & 37 \\
\hline SFA (\%E) & 7 & 7 & 7 & 7 & 7 & 6 & 6 & 7 & 6 & 8 & 6 & 8 & 7 & 8 & 7 & 7 & 7 & 7 \\
\hline PUFA (\%E) & 9 & 8 & 8 & 7 & 8 & 7 & 9 & 9 & 9 & 8 & 8 & 8 & 8 & 9 & 8 & 8 & 7 & 8 \\
\hline MUFA (\%E) & 9 & 9 & 9 & 9 & 9 & 9 & 9 & 11 & 9 & 11 & 9 & 11 & 9 & 12 & 9 & 11 & 9 & 11 \\
\hline Alcohol (g) & 0 & 0 & 0 & 0 & 0 & 0 & 0 & 0 & 0 & 0 & 0 & 0 & 0 & 5 & 0 & 5 & 0 & 5 \\
\hline Alcohol (\% energy) & 0 & 0 & 0 & 0 & 0 & 0 & 0 & 0 & 0 & 0 & 0 & 0 & 0 & 1 & 0 & 1 & 0 & 1 \\
\hline$P: S$ & $1 \cdot 27$ & $1 \cdot 18$ & $1 \cdot 20$ & $1 \cdot 12$ & $1 \cdot 13$ & $1 \cdot 19$ & $1 \cdot 35$ & $1 \cdot 20$ & 1.35 & $1 \cdot 12$ & $1 \cdot 29$ & 1.08 & $1 \cdot 19$ & $1 \cdot 12$ & $1 \cdot 19$ & $1 \cdot 12$ & $1 \cdot 13$ & $1 \cdot 12$ \\
\hline \multicolumn{19}{|l|}{ Thiamin (mg) } \\
\hline Mean & $1 \cdot 4$ & 1.5 & $1 \cdot 2$ & $1 \cdot 2$ & $1 \cdot 0$ & $1 \cdot 1$ & $1 \cdot 7$ & $2 \cdot 0$ & 1.5 & $1 \cdot 7$ & $1 \cdot 3$ & 1.5 & $1 \cdot 6$ & 1.9 & $1 \cdot 4$ & $1 \cdot 6$ & $1 \cdot 2$ & $1 \cdot 4$ \\
\hline \%RDI & 155 & 167 & 133 & 133 & 111 & 122 & 154 & 167 & 136 & 142 & 181 & 125 & 145 & 158 & 127 & 133 & 109 & 117 \\
\hline Riboflavin (mg) & & & & & & & & & & & & & & & & & & \\
\hline Mean & $2 \cdot 0$ & $2 \cdot 1$ & 1.9 & $2 \cdot 1$ & 1.9 & $2 \cdot 0$ & $2 \cdot 4$ & $2 \cdot 7$ & $2 \cdot 4$ & $2 \cdot 9$ & $2 \cdot 4$ & $2 \cdot 9$ & $2 \cdot 2$ & 2.9 & $2 \cdot 1$ & $2 \cdot 9$ & $2 \cdot 1$ & $2 \cdot 8$ \\
\hline$\%$ RDI & 222 & 233 & 211 & 233 & 211 & 222 & 218 & 208 & 218 & 230 & 218 & 230 & 200 & 223 & 191 & 223 & 191 & 215 \\
\hline Niacin equiv. (mg) & & & & & & & & & & & & & & & & & & \\
\hline Mean & 41 & 45 & 40 & 43 & 34 & 43 & 51 & 62 & 50 & 60 & 49 & 60 & 50 & 61 & 49 & 60 & 47 & 53 \\
\hline \%RDI & 342 & 375 & 333 & 358 & 283 & 358 & 365 & 385 & 357 & 375 & 350 & 375 & 357 & 381 & 350 & 375 & 394 & 331 \\
\hline Vitamin C (mg) & & & & & & & & & & & & & & & & & & \\
\hline Mean & 248 & 377 & 248 & 377 & 248 & 383 & 242 & 421 & 242 & 417 & 242 & 417 & 237 & 363 & 237 & 363 & 237 & 362 \\
\hline$\%$ RDI & 620 & 943 & 620 & 942 & 620 & 956 & 605 & 1053 & 605 & 1043 & 605 & 1043 & 527 & 807 & 527 & 807 & 527 & 804 \\
\hline Total folate $(\mu \mathrm{g})^{\star}$ & & & & & & & & & & & & & & & & & & \\
\hline Mean & 333 & 365 & 311 & 344 & 301 & 343 & 473 & 491 & 458 & 480 & 439 & 469 & 446 & 491 & 428 & 465 & 412 & 453 \\
\hline$\%$ RDI & 111 & 122 & 104 & 147 & 100 & 114 & 118 & 123 & 115 & 120 & 110 & 117 & 112 & 123 & 107 & 116 & 103 & 113 \\
\hline Total retinol equiv. ( & & & & & & & & & & & & & & & & & & \\
\hline Mean & 982 & 995 & 987 & 999 & 990 & 1177 & 1711 & 1644 & 1715 & 1662 & 1732 & 1667 & 1603 & 1649 & 1610 & 1654 & 1625 & 1659 \\
\hline$\%$ RDI & 163 & 166 & 165 & 167 & 165 & 196 & 244 & 183 & 245 & 185 & 247 & 185 & 229 & 183 & 230 & 184 & 232 & 184 \\
\hline $\mathrm{Na}(\mathrm{mg})^{\star}$ & $7451+$ & $7567+$ & $7496+$ & $7611+$ & $7376+$ & $7505+$ & $7891+$ & $10456+$ & $7891+$ & $10611+$ & $7784 t$ & $10483+$ & $7625+$ & $10552+$ & $7607 \dagger$ & $10557+$ & $7517+$ & $10450 t$ \\
\hline $\mathrm{K}(\mathrm{mg})$ & 3346 & 3880 & 3240 & 3774 & 3169 & 3763 & 4124 & 5031 & 4051 & 5003 & 3942 & 4924 & 3884 & 4945 & 3782 & 4830 & 3699 & 4751 \\
\hline $\mathrm{Mg}(\mathrm{mg})$ & & & & & & & & & & & & & & & & & & \\
\hline Mean & 465 & 513 & 395 & 443 & 361 & 416 & 547 & 671 & 504 & 610 & 435 & 574 & 525 & 670 & 480 & 597 & 413 & 560 \\
\hline \%RDI & 194 & 214 & 166 & 185 & 150 & 173 & 152 & 164 & 140 & 149 & 121 & 140 & 169 & 168 & 155 & 149 & 133 & 140 \\
\hline $\mathrm{Ca}(\mathrm{mg})$ & & & & & & & & & & & & & & & & & & \\
\hline Mean & 1103 & 1156 & 1102 & 1155 & 1089 & 1157 & 1406 & 1331 & 1405 & 1472 & 1402 & 1458 & 1193 & 1439 & 1189 & 1439 & 1191 & 1425 \\
\hline \%RDI & 110 & 116 & 110 & 116 & 109 & 116 & 108 & 102 & 108 & 113 & 107 & 112 & 119 & 144 & 119 & 144 & 119 & 143 \\
\hline $\mathrm{P}(\mathrm{mg})$ & & & & & & & & & & & & & & & & & & \\
\hline Mean & 1779 & 1877 & 1616 & 1715 & 1543 & 1649 & 2166 & 2339 & 2072 & 2352 & 1900 & 2274 & 1994 & 2492 & 1893 & 2325 & 1729 & 2246 \\
\hline \%RDI & 142 & 150 & 129 & 137 & 123 & 132 & 173 & 187 & 166 & 188 & 152 & 178 & 199 & 249 & 189 & 233 & 173 & 225 \\
\hline
\end{tabular}


lower in rice-based meal plans. Differences between $100 \%$ RG and $100 \%$ WG meal plans were minimised, with the major contributors to fibre being fruits and vegetables. To achieve the AI levels in the rice-based meal plans, careful consideration of the types of snack food and meal choices at breakfast, lunch and dinner was needed. The meal plans also consisted of a combination of different modern Westernised Asian foods drawn from many different Asian cultures.

The younger age groups were unable to achieve the four servings of grain-based foods daily while maintaining the recommended number of servings from other food groups, within the energy requirements. All meal plans, however, met or exceeded three servings of grainbased foods daily. One of the difficulties in including the four servings of grain-based foods within rice-based meal plans was the lack of grain-based snacks available. Snacks tended to be based on fruit or soya.

Creation of the meal plans focused on achievement of the nutrient targets as the primary goal secondary to food group targets. They were not created for institutional food service/food preparation purposes and hence some contained similar food choices on subsequent days. The plans were also limited to the foods contained within the available food composition databases at the time of the project. The AUSNUT $1999^{(16)}$ data did not contain the range of food choices presently available to the general public, nor a range of culturally specific foods. Many Asian food items needed to be added to the database (using food labels) as the AUSNUT database was derived from the previous National Nutrition Survey. This survey was conducted in 1995 during which time modern Asian cuisine was not as popular or available throughout Australia. The later NUTTAB 2006 database was also used to source some missing values. This database did contain a 'new' collection of Asian food items; however, many were still missing. The use of food packaging nutrient values was not ideal, but was required for the present study in the absence other suitable values.

The total $\mathrm{Na}$ values obtained for the rice-based meal plans did exceed the upper limit for $\mathrm{Na}$ (2300 mg for adults); however, limitations to food composition databases as discussed below suggest that these values may not be entirely accurate. The nutrient databases are also limited in their $\mathrm{Na}$ values. The 1999 data did not contain complete Na data, which if available would have seen further changes to total $\mathrm{Na}$ levels of the meal plans. The rice option in the database also only gave an option for rice cooked with salt. Steamed rice is popular among many Asian cultures, indicating again that the nutrient database was limited in its ability to adequately represent the food choices. The Australian food industry has also worked towards decreasing the $\mathrm{Na}$ content of foods over the past decade, also potentially decreasing the total values.

Similarly, the databases did not contain complete values for all food items for total folate, again potentially 
increasing this level. Similar limitations were also encountered for the breakdown of the nutrient data for specific fatty acids or for the inclusion of added or free sugars.

\section{Conclusion}

The results show that with careful food selection, meal plans with $\geq 4$ servings of grain-based foods daily can meet RDI and AI for lacto-ovo vegetarian and ricebased cuisines. Particular strategies to meet the nutrient needs for different ages and genders within each cuisine were required. The most important finding in terms of the difference between wholegrain and refined-grain foods was that a healthy individual's nutrient requirements could be met by including three to four servings of wholegrain or refined-grain foods daily.

\section{Acknowledgements}

Source of funding: This study was funded by an industry project supported by Go Grains Health \& Nutrition, Australia. Conflicts of interest: There are no conflicts of interest to declare. Authors' contributions: Y.P. was involved in the conduct of the research and drafting of the manuscript. L.T. provided advice during the research work and editing of the manuscript. Acknowledgements: The authors would like to thank Ms Holley Jones and Ms Sayne Dalton for their assistance with the study. The authors would also like to acknowledge Go Grains Health \& Nutrition for providing the funding for this project.

\section{References}

1. Smith AT, Kuznesof S, Richardson DP et al. (2003) Behavioural, attitudinal and dietary responses to the consumption of wholegrain foods. Proc Nutr Soc 62, 455-467.

2. Seal CJ (2006) Whole grains and CVD risk. Proc Nutr Soc 65, 24-34.

3. Dalton SMC, Tapsell L \& Probst Y (2012) Potential health benefits of wholegrain wheat components. Nutr Today $\mathbf{4 7}$, 163-174.

4. Steffen LM, Jacobs D, Stevens J et al. (2003) Associations of whole-grain, refined-grain, and fruit and vegetable consumption with risks of all-cause mortality and incident coronary artery disease and ischemic stroke: the Atherosclerosis Risk in Communities (ARIC) Study. Am J Clin Nutr 78, 383-390.

5. Liu S, Stamfer MJ, Hu FB et al. (1999) Whole-grain consumption and risk of coronary heart disease: results from the Nurses' Health Study. Am J Clin Nutr 70, 412-419.
6. Rangan A, Schindeler S, Hector DJ et al. (2009) Assessment of typical food portion sizes consumed among Australian adults. Nutr Diet 66, 227-233.

7. Lang R \& Jebb SA (2003) Who consumes whole grains, and how much? Proc Nutr Soc 62, 123-127.

8. National Health and Medical Research Council (2003) Food for Health: Dietary Guidelines for Australian Adults. A Guide to Healthy Eating. Canberra: Commonwealth of Australia; available at http://www.nhmrc.gov.au/_files_ nhmrc/publications/attachments/n29.pdf.

9. Marquart L, Salvin JL \& Fulcher RG (2002) Whole-grain Foods in Health and Disease. St. Paul, MN: American Association of Cereal Chemists, Inc.

10. Smith A, Kellet E \& Schmerlaib Y (1998) The Australian Guide to Healthy Eating. Background Information for Nutrition Educators. Canberra: Commonwealth of Australia.

11. Messer E (1989) Methods for studying determinants of food intake. In Research Methods in Nutritional Anthropology, pp. 1-33 [G Pelto, P Pelto and E Messer, editors]. Tokyo: United Nations University.

12. National Institute of Health and Welfare (2009) Vegetarian diet. http://www.nlm.nih.gov/medlineplus/vegetariandiet. html (accessed January 2009).

13. Australian Government Culture Portal (2008) Australian food and drink. http://www.cultureandrecreation.gov.au/ articles/foodanddrink/ (accessed November 2008).

14. Heinemann RJB, Fagundes $\mathrm{Pl}$, Pinto EA et al. (2005) Comparative study of nutrient composition of commercial brown, parboiled and milled rice from Brazil. J Food Compost Anal 18, 287-296.

15. Department of Health and Ageing, National Health and Medical Research Council (2006) Nutrient Reference Values for Australia and New Zealand. Canberra: Australian Government.

16. Australia New Zealand Food Authority (1999) AUSNUT Australian Food and Nutrient Database. Canberra: ANZFA.

17. National Health and Medical Research Council (2006) Nutrient Reference Values for Australia and New Zealand: Executive Summary. Canberra: NHMRC.

18. Australian Bureau of Statistics (1995) How Australians Measure Up. Catalogue no. 4359.0. Canberra: ABS.

19. Department of Education and Early Childhood Development, State Government of Victoria (2008) Weight: child health record: growth details. http://www.education.vic.gov. $\mathrm{au} /$ ecsmanagement/mch/childhealthrecord/growth/default. htm (accessed November 2008).

20. Food Standards Australia New Zealand (2007) NUTTAB 2006 online version. http://www.foodstandards.gov.au/monitoringandsurveillance/nuttab2006/onlineversionintroduction/ onlineversion.cfm (accessed October 2008).

21. US Department of Agriculture, Nutrient Data Laboratory (2008) USDA Nutrient Database for Standard Reference; Release 21. http://www.nal.usda.gov/fnic/foodcomp (accessed October 2008).

22. Vesa T, Marteau P \& Korpela R (2000) Lactose intolerance. J Am Coll Nutr 19, 2 Suppl., 165S-175S.

23. Bhat R, Deosthale Y, Roy D et al. (2006) Nutritional and toxicological evaluation of black tip rice. J Sci Food Agric 33, 41-47. 